\title{
A propos du partage du potentiel hydro-électrique dans les aménagements de cours d'eau communs à plusieurs états
}

\author{
PAR
}

\author{
Vlad Focsa \\ Ingénieur conseil, diplömé de l'Eeole Polytechnique Fédérale de Zïrich, \\ Chef du projet de l'aménagement des Portes de Fer. sur le Danube. \\ Ancien expert des Nations-Unies
}

\section{Introduction}

La mise en valeur du potentiel hydro-électrique des cours d'eau traversant plusieurs états (en série) ou formant frontière entre deux états (contigus) soulève souvent des problèmes difficiles à résoudre, quant au partage entre les riverains des avantages apportés par les ouvrages projetés : production d'énergie électrique, utilisation des débits régularisés.

En raison de cette difficulté, un très petit nombre de fleuves importants, communs à plusieurs pays, ont été aménagés jusqu'à présent et l'utilisation des ressources hydrauliques de ces cours d'eaux n'a pas fait l'objet d'études générales $\left({ }^{1}\right)$.

Pour les cours d'eau internationaux, la littérature technique ainsi que les Nations Unies recommandent d'adopter des schémas d'aménagements intégrés ne tenant pas compte des frontières et permettant une optimisation d'ensemble. Les riverains sont certainement intéressés à choisir un schéma apportant le maximum d'avantages, cependant, souvent l'optimum général ne coïncide pas avec l'ensemble des optima de chaque partenaire.

Les projets d'aménagement hydraulique des cours d'eau à débits importants comportent en général une chaîne de barrages avec des usines hydro-électriques en cascade. On tend à adopter entre les biefs des hauteurs de plus en plus réduites ( 6 à $16 \mathrm{~m}$ ), afin de limiter les superficies inondées et les dégâts dans la zone du remous. Il s'agit donc d'usines au fil de l'eau, à faible accumula-

(1) U.N. Integrated River Basin Development N.I. 1970 E/3066 tev. 1 tion, capables tout au plus de modulations journalières et hebdomadaires. Pour ces petites hauteurs, les barrages ne présentent pas, en général, de difficultés de construction et peuvent être placés pratiquement à n'importe quelle section du fleuve, à condition de prévoir à l'amont, le long des berges, des digues et des systèmes de drainage, pour protéger les terrains riverains contre les effets du remous. Les digues de protection, de hauteur modeste, n'exigent pas de très grands investissements, surtout si l'exploitation de l'usine s'effectue à niveau variable : niveau au barrage baissé pendant les crues afin de réduire le remous en amont, et relevé aux étiages pour accroître la hauteur de chute $\left({ }^{2}\right)$.

La grande latitude de choix des emplacements des barrages conduit à une multitude de variantes de sché-

(2) Sur les cours d'eau importants, les remous créés par les barrages doivent être déterminés avec beaucoup de soin à partir de points de contrôle du niveau (limnimètres) en amont, afin de permettre une exploitation de l'usine jusqu'à la cote maximale possible, sans toutefois dépasser les niveaux maximaux autorisés tout le long de la retenue. C'est une opération extrêmement délicate car les niveaux dans la retenue sont influencés, d'un côté par les mouvements non permanents provoqués par les variations rapides du niveau au barrage (dues surtout aux éclusées) et d'un autre côté par les variations lentes de niveau dues à la variation des débits affluents. L'ensemble de ces mouvements irréguliers peut faire l'objet d'analyse sur modèle mathématique en vue de fixer à tout instant la cote maximale d'exploitation.

Dans un aménagement d'un grand fleuve d'Europe dont le débit moyen est $5500 \mathrm{~m}^{3} / \mathrm{s}$, chaque décimètre de chute supplémentaire représente $45 \mathrm{GWh} / \mathrm{an}$. Dans l'étude du projet d'usine hydroélectrique de cet aménagement, le calcul du remous comporte l'utilisation de caractéristique physique de sections transversales distantes de 3 à $4 \mathrm{~km}$. Dans chaque section, un limnimètre a été installé sur chaque berge. L'observation journalière de ces appareils pendant une période de 4 ans a permis d'obtenir pour une large gamme des débits les profils du fleuve sur les $200 \mathrm{~km}$ de longueur du remous. 
mas d'aménagement pour un tronçon de cours d'eau. Par exemple, l'étude des $1075 \mathrm{~km}$ du Bas-Danube, du Delta à la frontière roumano-yougoslave (Bazias) faite après reconnaissances in situ prenait finalement en considération 17 emplacements de barrage avec chacun des hauteurs diverses, conduisant au total à 64 variantes de schémas d'aménagement pour cette partie du fleuve. Les écarts entre les valeurs de production totale d'énergie électrique obtenues dans les meilleurs de ces schémas se sont relevés minimes ; toutefois les avantages revenant à chacun des quatre états riverains (Yougoslavie, Roumanie, Bulgarie et Union Soviétique) différaient considérablement d'un schéma à l'autre.

\section{Principes de droit relatifs à l'aména- gement des eaux internationales}

Le droit international public ne comporte pas de principes et de dispositions unanimement reconnus en matière d'aménagement des cours d'eau internationaux, soit contigus, soit successifs. De nombreux essais pour parvenir à des règles communes, ont été tentés à partir de l'interprétation des règles générales du droit international, de l'étude de la jurisprudence, de l'analyse des différentes pratiques contenues dans les conventions conclues exprimant les positions officielles des juristes, ou des gouvernements en cause, de l'examen des ouvrages juridiques des spécialistes, et enfin des conférences internationales, notamment celles de l'Institut de droit international, de la Ligue des Nations et des Nations Unies. On constate des interprétations totalement opposées des principes de droit, d'abord chez les spécialistes du droit international mais surtout, dans les positions soutenues par les états, qui cherchent dans chaque cas à défendre leurs intérêts politiques, économiques, stratégiques, etc. . .

Mème en recherchant des principes universels, avec la meilleure bonne foi, on constaterait que le problème de l'aménagement des cours d'eau est extrêmement complexe et contient des aspects en évolution permanente, ou non encore précisés, impossibles à saisir par des règles générales.

Toutefois, de grands efforts ont été faits pour dégager quelques principes qui pourraient servir de guide, ou au moins aider les parties intéressées à trouver une solution.

En 1911 à Madrid, une réunion de l'Institut de droit international a adopté une Déclaration concernant la réglementation internationale de l'usage des cours d'eau internationaux. L'idée dominante est l'obligation pour un état d'obtenir l'assentiment préalable des autres états riverains avant d'entreprendre des travaux sujets à modifier le régime hydraulique du fleuve ou à provoquer des préjudices aux autres états.

Patronnée par la Ligue des Nations, une Conférence générale des communications et du transit s'est réunie à Barcelone (1921) et a poursuivi ensuite ses travaux à Genève (1923-1939). Beaucoup d'idées y ont été exprimées, sans toutefois aboutir à des principes unanimes.

Une Sous-Commission de cette Conférence a été créée en 1922 à Genève, chargée des questions relatives aux aménagements hydro-électriques. La législation en matière de droit des eaux dans les différents états et dans les conventions internationales conclues" lors d'aménagements hydro-électriques présente de grandes différences. Aussi, la Sous-Commission a-t-elle élaboré en 1923 une "Convention-cadre relative à l'aménagement des forces hydroélectriques intéressant plusieurs états" dans le but de promouvoir le développement de l'utilisation des ressources potentielles des rivières, de faciliter les accords entre les états dans ce domaine et de protéger les états -surtout ceux situés à l'aval- contre les préjudices qui en résulteraient.

Cette convention-cadre contenait l'obligation pour les autres états de se mettre d'accord, si un état se proposait d'entreprendre la réalisation d'un aménagement hydroélectrique sur un cours d'eau international. L'application de cette disposition était en outre contrôlée par la Ligue des Nations, qui apparaissait donc comme un super-état, C'est essentiellement l'inclusion de ce principe dans la convention qui a fait que très peu d'états l'ont ratifiée.

Les idées directrices des travaux effectués à ce propos peuvent se résumer comme suit :

\section{a) Tracé de la frontière}

La pratique établit que pour les cours d'eau navigables le tracé de la frontière est représenté par le chenal navigable (Thalweg, Fahrwasser). Or, à la suite de crues, même pour les grands fleuves, le chenal navigable peut subir des changements, passer d'un bras à l'autre du fleuve ; le chenal lui-même a une largeur variable tandis que la frontière est une ligne précise. Aussi, beaucoup d'états ont défini géodésiquement par des repères sur les deux rives, consignés par des protocoles communs, la ligne de frontière, dans le lit des cours d'eau.

Pour les rivières qui ne sont pas navigables, la frontière est considérée généralement comme étant la ligne médiane entre les berges pendant les étiages ; mais comme le lit des rivières est variable, les lignes de frontière dans les lits des rivières ont été marquées en général en même temps que la frontière terrestre.

Quoique le tracé de la frontière dans le lit des cours d'eau ne paraisse pas donner matière à polémiques, les juristes lui accordent une importance majeure, liée à l'intégrité et à l'inaliénabilité du territoire, en tant que principe inscrit dans les constitutions de tous les états. Ainsi, même après la construction d'un barrage qui change complètement le contour des berges à l'amont, la frontière ne change pas et des dispositions géodésiques doivent permettre de la retrouver après travaux $\left({ }^{3}\right)$.

\section{b) Ordre d'importance des utilités de l'eau}

L'utilisation des ressources hydrauliques des cours d'eau intéresse divers secteurs de la vie économique. Au siècle dernier, la navigation, au moins dans les régions humides, constituait l'utilisation prioritaire $\left({ }^{4}\right)$. L'essor de l'énergie

(3) Aux Etats-Unis, la navigation a une priorité de par la Constitution.

(4) Par la loi fédérale relative au Colorado (1928), l'odre suivant d'importance est établi : protection contre les crues et navigation eau potable et irrigation; énergie hydroélectrique.

Par le Traité de 1944 entre les Etats-Unis et le Mexique relatif á l'usage en commun des eaux internationales, un autre ordre d'importance est prevu : usages domestiques et municipaux : agriculture et élevage ; énergie hydro-électrique ; autres usages industriels ; navigation ; pêche ; autre utilisations. 
hydroélectrique, a été lié au développement industriel et dernièrement, la hausse spectaculaire des prix des combustibles lui a donné un regain d'intérêt. Mais l'accroissement de la population confère maintenant une place toujours plus importante à l'irrigation et à l'approvisionnement en eau pour l'agriculture. L'urbanisation qui conduit à des agglomérations toujours plus concentrées, crée en même temps des problèmes pour leur alimentation en eau. Les industries toujours plus puissantes nécessitent des besoins en eau de plus en plus grands. Enfin, la protection contre les inondations, qui est une responsabilité permanente de chaque état, reste une préoccupation majeure dans chaque projet d'aménagement des cours d'eau.

En définitive pour chaque rivière, les utilités prioritaires sont liées à la conjoncture. Tout projet d'aménagement doit prendre en considération les finalités présentes et futures dans un schéma général permettant la justification économique des solutions présentées du point de vue de l'intérêt public. Il y a lieu de tenir compte éventuellement des droits acquis pour les finalités anciennes pouvant donner lieu à indemnisation si elles ne sont plus susceptibles d'être envisagées dans le projet.

\section{c) Souveraineté et propriété sur les eaux internationales}

Le droit qu'a chaque état riverain sur les débits des rivières internationales étant primordial, le problème se pose de la mise en valeur et du partage entre les riverains des différentes ressources hydrauliques. Dans les pays secs ou demi-arides, l'eau ne suffit jamais et son partage est par conséquent très disputé. Dans les pays humides, même si actuellement on peut imaginer des solutions pour le partage des débits, les gouvernements hésitent à prendre des options pour l'avenir. Aussi, s'il existe actuellement beaucoup de conventions pour le partage du potentiel hydroélectrique concernant des usines communes à plusieurs états, il y a très peu d'accords sur le partage des débits des cours d'eau internationaux.

Les principes de droit en la matière sont aussi opposés que possible, allant de la "souveraineté absolue", (un état pouvant user à discrétion des ressources hydrauliques autant que le cours d'eau coule sur son territoire, même en consommant tout le débit, sans solliciter aucun assentiment des corriverains), à l'obligation de ne pas entreprendre des travaux qui pourraient modifier le régime existant de la rivière, sans l'accord préalable des corriverains -donc une "souveraineté limitée"-. L'idée de souveraineté limitée est un non sens en soi : la souveraineté existe et elle est totale, c'est-à-dire que dans les limites de son territoire l'état exerce son autorité sur les personnes et les choses qui s'y trouvent. Cependant, même sans renoncer à ce principe, on peut toujours convenir par des compromis, d'arrangements $\left(^{5}\right)$ pour aboutir à des solutions communes.

(5) Ùn exemple d'un pareil Arrangement, cité par la publication E/ECE/EP/98-1952 des N.U. Commission Economique pour l'Europe "Aspects juridiques de l'aménagement hydro-électrique des fleuves et des lacs d'intérêt commun" est l'Accord conclu en 1923 entre la Bavière et l'Autriche, prorogé en 1947 par un Accord entre les gouvernements militaires Britannique et Américain et la République d'Autriche, relatif aux eaux d'intérêt commun :

En vertu des principes généraux de droit, chaque état peut prétendre à l'utilisation de la moitié des débits sur le parcours d) Pratiques juridiques régissant les aménagements hydroélectriques des eaux internationales

$\mathrm{Vu}$ qu'en cette matière, il n'existe pas de principes de droit unanimement admis, on a essayé de tirer des débats de diverses commissions de droit international, ainsi que de la pratique utilisée dans les conventions passées pour la réalisation d'usines hydro-électriques communes à plusieurs états, un certain nombre d'idées directrices de nature à faciliter les solutions dans ce genre de problèmes :

1) Chaque état a, sur la partie de rivière coulant sur son territoire, droit de souveraineté et de propriété. En conséquence, le droit de construire les ouvrages en rivière qu'il estime nécessaires pour la mise en valeur de son potentiel hydro-électrique lui appartient pleinement.

2) L'état concerné doit veiller à ce que les modifications au tracé et au régime existant du fleuve consécutives aux installations réalisées et à leur exploitation, ne provoquent pas de préjudices aux corriverains.

Pour les rivières "contiguës", l'aménagement d'une partie du lit du fleuve entrainant forcément des répercussions sur l'autre rive doit ètre subordonné à l'accord des corriverains.

Un état peut aménager unilatéralement le secteur d'un cours d'eau (en série) traversant son territoire, pourvu que la zone d'influence aval et amont de cet aménagement n'entraine que des dommages restreints aux riverains des autres états.

La défïition de ce que représente un préjudice restreint ou grave étant difficile, les états intéressés négocieront l'évaluation des dédommagements (très souvent ceux-ci consistent en livraison d'énergie électrique).

Un principe nouveau qui tend à s'affirmer est de s'assurer que même dans le cas limite de la détérioration, ou même de la destruction totale des installations (suite à un tremblement de terre, avarie grave ou tout autre cause), les riverains d'aval ne seront pas exposés à des effets catastrophiques.

3) Les relations internationales actuelles sont marquées par un esprit de coopération, d'équité et de bon voisinage entre les nations, reposant sur le respect des intérêts de chaque état et de l'égalité dans les tractations. Dans ce climat, un état ne pourrait pas s'opposer à la réalisation d'un aménagement qu'un corriverain désire réaliser ; par des négociations, on recherchera une solution objective conciliant les intérêts de chaque partie.

La pénurie d'énergie et la hausse récente des prix des combustibles liquides qui a remis en vedette les aménagements hydro-électriques sont de nature à faciliter les arrangements pour leur réalisation.

4) La pratique usuelle est de débuter toute négociation par l'établissement d'une commission gouvernementale mixte dont la caractéristique est d'être à la fois économique, juridique, et bien-entendu politique.

contigu des cours d'eau,

- Pour les rivières successives, l'Autriche maintient le droit de souveraineté territoriale d'après lequel un pays a à sa disposition entière le débit coulant sur son territoire.

Toutefois, sans renoncer à ce principe juridique, elle serait disposée en vue d'une exploitation plus avantageuse, à communiquer à la Bavière les projets d'aménagement qu'elle a en vue, sous réserve de réciprocité et d'examiner les observations sur ces projets, sans toutefois s'engager de les accepter. 
La commission mixte intervient dans les études et l'élaboration des projets, elle les approuve et elle prépare les traités, accords, conventions ou protocoles nécessaires pour la construction et l'exploitation des aménagements projetés $\left({ }^{6}\right)$.

Les Conventions traitent aussi des différents qui pourraient intervenir dont les solutions sont généralement confiées à la Cour permanente de Justice.

5) Les nouveaux accords doivent prendre en considération les anciennes conventions bi et multilatérales entre riverains sur la navigation, la pêche, etc. .

\section{Le partage du potentiel hydro- électrique des cours d'eau communs}

L'énergie hydro-électrique brute d'un cours d'eau est définie par :

$$
E=\int_{0}^{T} k Q H^{d t}
$$

où $Q$ est le débit (en $\mathrm{m}^{3} / \mathrm{s}$ ), $H$ est la différence entre les niveaux de l'eau à l'entrée et à la sortie du secteur de rivière utilisé (en $\mathrm{m}$ ), $K$ est un coefficient qui dépend des conditions physiques du lit de la rivière, $T$ est la période de temps (en général, une année, soit 8760 heures).Les valeurs $K, Q$ et $H$ sont variables dans le temps et en interdépendance. L'expression 1 est intégrée sur une période hydrologique représentative. Toutefois, souvent l'évaluation de l'énergie potentielle est faite en utilisant le débit moyen sur plusieurs années et la différence de niveau de l'eau qui résulte de ce débit entre l'entrée et la sortie du secteur aménagé.

Sur les grands fleuves, un barrage, même de hauteur réduite (inférieure à $15 \mathrm{~m}$ ) provoque un remous qui s'étend sur de grands parcours. Sur les cours inférieurs des fleuves où les débits sont importants et les pentes modestes, les remous peuvent se propager souvent sur des distances de plusieurs centaines de $\mathrm{km}$. La longueur du remous varie avec le débit, diminuant avec les crues, augmentant aux étiages. Plus les remous sont longs, plus les pertes de potentiel hydro-électrique sur son parcours sont importantes, cf. fig. $2: H-(\mathrm{Ham}+\mathrm{Hav})$.

Théoriquement, les remous se font ressentir par de très petites dénivellations, loin à l'amont. Afin de fixer un point d'arrêt $\mathrm{C}$, il faut convenir d'une tolérance sur cette dénivellation; dans la pratique, on prend de 10 à $25 \mathrm{~cm}$ en débits moyens.

Si le secteur de rivière aménagé est commun sur toute sa longueur, le problème du partage du potentiel est aisé, à chaque état revenant la moitié de la puissance de l'énergie électrique produite. Toutefois, le partage peut être lié à la

(6) Un exemple en la matière a été la Commission Gouvernementale mixte roumano-yougoslave pour l'aménagement du Danube aux Portes de Fer, qui a préparé l'Accord général pour la réalisation de cet ouvrage, ainsi que les conventions annexes pour : l'élaboration des projets, l'exécution des ouvrages, l'exploitation des aménagements, la détermination des coûts d'investissements et le décompte réciproque, l'estimation des dégâts et des dédommagements, la modification de la frontière, le franchissement de la frontière durant les travaux, etc. . . L'accord et les conventions ont été publiés dans le "Recueil des traités" - U.N. Treaty Series vol. 512 et 513 participation des deux états aux coûts d'investissement, voire à d'autres éléments qui peuvent ne pas être équivalents sur les deux rives.

Dans le cas où seulement le secteur $\mathrm{AB}$ est commun et le secteur $\mathrm{BC}$ appartient entièrement à un des états, le partage du potentiel soulève souvent des difficultés. Les recommandations de la littérature technique ou didactique et la pratique de beaucoup de conventions conclues $\left({ }^{7}\right)$ considèrent équitable le partage de l'énergie dans la proportion du potentiel hydro-électrique, appartenant à chacun des deux états, soit :

$$
\begin{gathered}
E_{1}=\int_{o}^{T} K Q\left(\frac{H a v}{2}+H a m\right) d t \\
E_{2}=\int_{0}^{T} K Q \frac{H a v}{2} d t
\end{gathered}
$$

Il est évident qu'un partage du potentiel suivant une formule (cf. fig.)

$$
\begin{gathered}
E_{1}=\int_{o}^{T} K Q\left(\frac{H a v}{2}+H-H a v\right) d t \\
E_{2}=\int_{o}^{T} K Q \frac{H a v}{2} d t
\end{gathered}
$$

est aberrante car la somme surpasse le potentiel théorique utilisable de l'aménagement projeté.

Par ailleurs, pour les deux riverains, chaque position du barrage et chaque cote de rétention représente un degré différent (plus ou moins favorable) d'utilisation effective du potentiel théorique de son secteur de rivière (variantes I et II de la fig.).
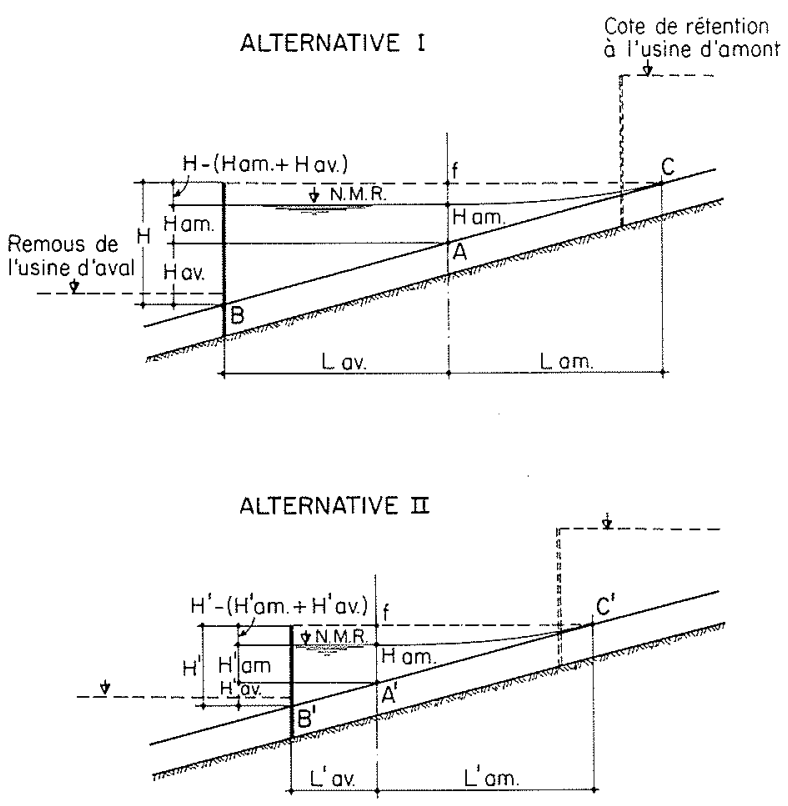
$\begin{aligned} F f= & \text { frontière d'état ; le secteur commun du cours d'eau } \\ & \text { peut être soit à l'amont du point } f \text {, soit a l'aval. }\end{aligned}$

(7) Cette formule de partage a été souvent admise : l'Allemagne et la Suisse l'ont utilisée pour partager le potentiel du Rhin aux usines hydro-électriques communes installées entre le Lac de Constance et Bâle, où la frontière ne suivait pas strictement le fleuve. Un autre exemple est le partage entre la Suisse et l'Italie du potentiel aménagé par l'usine Inner Ferera en proportion de $70 \%$ et $30 \%$ compte tenu des débits et de la chute utilisés sur chaque territoire. 
En effet, il est possible que, même si le schéma par la variante I offre un effet énergétique total supérieur $(H a v+$ $H(a m)>\left(H^{\prime} a v+H^{\prime} a m\right)$ à la variante II, un des riverains obtient $\left(\frac{H^{\prime} a v}{2}+H^{\prime} a m\right)$ de son secteur de rivière plus d'énergie par le schéma II, en déplaçant le barrage vers l'amont. La solution est très malaisée, car pour un des partenaires, les différences des avantages entre les différentes variantes sont souvent considérables. Pour rechercher une solution, les partenaires étudieront, soit en commun, soit chacun pour soi, maintes variantes d'emplacement du barrage et de sa cote de retenue. Outre la production d'énergie électrique, les intérêts d'autres secteurs économiques, le coût d'installation, et d'autres éléments peuvent entrer en jeu. Au vu des résultats de telles études, on pourra définir par négociations la section d'implantation du barrage et la cote de retenue, au besoin à l'aide d'un compromis conciliant les intérêts des deux états.

Dans beaucoup de cas, aucun des partenaires ne souhaite être minoritaire dans l'opération à réaliser et si matériellement d'après la formule 2 , il ne lui revient pas la moitié des avantages, il cherche par des compensations de différentes natures à arriver à la parité de participation dans l'aménagement commun.

Un exemple d'arrangements dans ce sens a été l'accord conclu pour l'usine hydro-électrique Jochenstein sur le Danube $\left({ }^{8}\right)$. La frontière entre l'Autriche et la République Fédérale d'Allemagne est sur la rivière Inn jusqu'à son confluent avec le Danube, suit ensuite le cours du Danube sur $20 \mathrm{~km}$ en aval de ce confluent et continue sur un tracé perpendiculaire au fleuve. Le potentiel hydro-électrique des secteurs communs et de l'Inn appartient paritairement aux deux états. L'étude de l'utilisation de l'énergie hydroélectrique de ces deux cours d'eau, faite dans le schéma général d'utilisation des ressources hydrauliques du fleuve, en tenant compte de la morphologie, des conditions géologiques, des possibilités rationnelles d'inonder les zones riveraines, ainsi que de l'amélioration des conditions de navigation indiquaient comme emplacement du barrage optimum sur le Danube, Jochenstein, peu à l'amont de la fin du secteur commun du fleuve. Il restait ainsi inutilisé sur le secteur commun du Danube le potentiel entre Jochenstein et la fin du secteur commun. Cependant, la cote de retenue qui permettait la conjugaison favorable avec l'usine existante de Kachlet en amont sur le fleuve. en secteur allemand, incluait l'utilisation du potentiel exclusivement allemand du fleuve, compris entre le confluent Inn/Danube et Kachlet.

Afin d'arriver à un partage rationnel du potentiel, des négociations poursuivies entre 1949-1952 aboutirent à un arrangement prévoyant le dragage du lit du fleuve sur une longueur de $7000 \mathrm{~m}$ en aval de Jochenstein, sur une profondeur moyenne de 1,2 m. Par ce moyen, la hauteur de chute aux turbines était accrue, et l'usine de Jochenstein utilisait le potentiel du fleuve jusqu'à la fin du secteur aval commun avec, en plus une partie du potentiel d'aval exclusivement autrichien, équivalent au potentiel exclusivement allemand d'amont.

Les travaux ayant été exécutés suivant cette conception l'usine de Jochenstein a une puissance installée de

(8) V. Focsa et C. Borc, L'excavation du lit du Danube à l'aval du barrage des Portes de Fer, Hidroenergetica, vol. 15 - août 1970
$145 \mathrm{MW}$ et une production annuelle d'énergie électrique de $920 \mathrm{GWh}$, chaque pays étant partenaire à parts égales.

Un arrangement semblable et dans le même but avait été traité entre l'Autriche et la Tchécoslovaquie pour la construction en commun de l'usine hydroélectrique de Bratislava sur le Danube (km. D 1873), mais pour diverses raisons, l'ouvrage n'a pas pu être réalisé.

Un autre exemple d'arrangement analogue est l'aménagement des Portes de Fer sur le Danube $\left({ }^{9}\right)$ : le remous du barrage (situé au km. D. 942,95), dépasse le secteur commun roumano-yougoslave, qui s'arrête au confluent avec la rivière Nera $(\mathrm{km}(\mathrm{km} \mathrm{D.} \mathrm{1075)} \mathrm{et} \mathrm{s'étend} \mathrm{encore}$ sur environ $120 \mathrm{~km}$, jusque dans la région de Belgrade, utilisant environ $6,5 \mathrm{~m}$ de chute de ce dernier secteur, entièrement yougoslave $(1 \mathrm{~m}$ de chute sur ce secteur du Danube représente une production annuelle d'énergie d'environ $450 \mathrm{GWh}$ ). Un accord a pu être conclu entre les deux pays, établissant le partage a parité de la puissance installée de $2100 \mathrm{MW}$ et de la production d'énergie de $11000 \mathrm{GW} / \mathrm{an}$, Cependant, en compensation, la Roumanie participait avec 20 millions US $\$$ aux travaux de régularisation de la rivière Norava (située en territoire yougoslave); ces travaux permettent de retenir une partie importante des alluvions déversées en abondance par cette rivière dans la retenue des Portes de Fer.

Le partage du potentiel hydroélectrique est couramment lié à la participation des partenaires aux coûts d'investissements de l'ouvrage projeté. Les éléments de coût (prix des matériaux, main-d'oeuvre, équipements, taux des dédommagements $\left({ }^{10}\right)$, prix de vente et débouché pour l'énergie produite) dépendent de la conjoncture; il y a lieu de tenir compte de leur évolution sur la période de construction et celle du remboursement des crédits. Enfin, les deux états peuvent avoir des priorités dans leurs programmes d'équipement hydro-électrique qui ne s'inscrivent pas dans les mêmes périodes de temps, et si l'intérêt est très actuel pour un des partenaires, il ne l'est pas forcément aussi pour l'autre.

Dans ces conditions, bien que le potentiel hydroélectrique ait un caractère de permanence et de propriété exclusive des états riverains, son partage suit de plus en plus rarement la règle des autres secteurs économiques, qui parfois s'entravent mutuellement, y sont mêlés. Donc ce partage qu'on pourrait désigner plutôt comme taux de participation aux coûts d'investissements, ainsi qu'aux bénéfices de l'aménagement est toujours négocié. Il est toutefois évident que, puisque l'objet des tractations -le potentiel hydro-électrique - est d'abord un bien national à caractère inaliénable, les écarts à la règle 2 devront être justifiés avec soin et beaucoup d'objectivité, les accords réalisés restant valables pour de très longues périodes.

Enfin, il faut mentionner un autre élément sensible de plus en plus évoqué dans les accords : celui de la propriété des ouvrages. Chaque partenaire voudrait avoir sa centrale électrique sur son territoire propre. Dans ce but on est souvent conduit à rejeter la meilleurs solution technique de disposition générale des ouvrages, même si cela

(9) V. Focsa et Fl. Iorgulescu, L'aménagement complexe du Danube aux Portes de Fer, Travaux $n^{\circ}$ 434, mai 1971.

(10) La zone d'influence du barrage peut être ressentie même à l'amont du remous par des atterrissements qui remontent hors de ces limites (remous solide) et qui entrainent des dépenses, parfois importantes, pour rétablir la situation. 
entraîne des frais supplémentaires, ou alors on procède par rectification de frontière.

\section{Cas de trois états ziverains}

Si le secteur de rivière aménagé comporte une berge sur le territoire d'un état et l'autre berge sur celui de deux autres états, il y aura trois partenaires pour cet aménagement. Le premier ne pourra pas prétendre simplement à la moitié de l'énergie électrique produite, car les investissements pour la réalisation des ouvrages, notamment ceux concernant la protection des territoires riverains et les indemnisations ne sont pas forcément a parité sur les deux rives. Les taux de partage de l'énergie sont évidemment liés à la participation des partenaires aux coûts de la construction. Le premier partenaire pourrait, par exemple, adopter comme position de prétendre à la moitié de l'énergie produite et de supporter la moitiè de la totalité des dépenses. Les deux partenaires de l'autre rive, de leur côté, pourraient soutenir que pour chaque rive la mise en valeur du potentiel est lié à des conditions spécifiques d'indemnisation, que les riverains respectifs doivent supporter chacun pour son territoire. Les deux positions peuvent présenter parfois des différences considérables en ce qui concerne les sommes à débourser et il est évident qu'une solution ne pourra pas être trouvée par l'abandon d'un des principes, mais par un compromis entre les partenaires.

Afin de déterminer la hauteur Ham (cf. fig.) qui détermine le potentiel utilisé appartenant au partenaire d'amont, une analyse technique et économique très détaillée est à effectuer.

La cote de retenue à l'usine située à l'aval devra être déterminée par un calcul d'optimisation de la production d'énergie électrique de l'ensemble des usines aval et amont tenant compte aussi de la qualité de l'énergie obtenue. (La tendance est de produire par des éclusées journalières et souvent bijournalières le maximum d'énergie de pointe de valeur bien supérieure (11)).

Par ailleurs, les riverains sont tentés de prévoir l'emplacement du barrage le plus en aval du secteur commun de la rivière, afin d'utiliser le maximum de leur potentiel dans des usines situées sur leurs territoires.

Après la construction de l'usine, le lit de la rivière subit en aval des érosions sur des profondeurs importantes, parfois de plusieurs mètres. Ce phénomène est dû principalement à la clarification de l'eau du fleuve qui dépose les alluvions en suspension dans la retenue d'amont, mais aussi aux effets provoqués par le déversement des crues et l'exploitation de l'usine hydroélectrique en éclusées.

Ces érosions peuvent avoir des conséquences bénéfiques et maléfiques diverses, qui seront à analyser, mais du point de vue énergétique, la chute utilisée aux turbines augmente par l'utilisation d'une différence de niveau supplémentaire qui appartient aux riverains d'aval. L'utilisation de ce supplément de potentiel doit être consentie par ces riverains d'aval -qui peuvent être un ou deux autres étatsmoyennant bien entendu une juste compensation (12).

\section{Conclusions}

Le potentiel hydroélectrique d'une rivière, formant sur une partie de son cours la frontière entre deux ou plusieurs états, appartient aux états riverains respectifs.

Pour l'étude d'utilisation du potentiel hydro-électrique, la littérature technique recommande d'adopter un schéma d'aménagement de la rivière entière, optimal du point de vue énergètique, sans tenir compte des frontières d'état ; le partage entre les riverains des effets obtenus (puissance installée, production d'énergie électrique) s'effectuera proportionnellement au potentiel utilisé par les différentes usines hydro-électriques du schéma appartenant à chaque état.

Cependant, dans beaucoup de cas, du fait de divers éléments autant techniques qu'économiques ou politiques, l'application de ce principe de partage ne couvre pas favorablement les intérêts spécifiques de chacun des états participants. La solution de partage du potentiel, dans ces cas, est recherchée par des négociations visant un arrangement acceptable pour les parties en cause.
(11) Sur les rivières navigables, l'ampleur des éclusées n'est limitée que par la condition d'assurer en aval de l'usine le tirant d'eau prescrit.
(12) Par exemple, par des livraisons d'énergie électrique del'usine en cause (quantité d'énergie à livrer en tenant compte aussi des coûts d'établissement et exploitation de l'usine). 